\title{
Soluciones basadas en la Naturaleza: estrategias urbanas para la adaptación al cambio climático
}

\author{
Nature based Solutions: urban strategies \\ for adaptation to climate change
}

\author{
Raquel Rey Mellado, ${ }^{1}$ Cristina del Pozo Sánchez ${ }^{2}$ y María Teresa Franchini Alonso ${ }^{3}$
}

Fecha de recepción: 30-01-2021 - Fecha de aceptación: 05-08-2021

Hábitat y Sociedad (ISSN 2173-125X), n. ${ }^{\circ}$ 14, noviembre de 2021, pp. 243-262.

https://doi.org/10.12795/HabitatySociedad.2021.i14.13

\section{Summary}

Cities will suffer the impacts of climate change in the next decades. These impacts will be different according to their geographical features, the distribution and number of green spaces, the characteristics of the exterior surfaces of their floors and the density of population, among other aspects.

Given this situation, many cities have begun to adopt adaptation strategies to reduce their vulnerability to the adverse effects of the climate; among which $\mathrm{Na}$ ture-Based Solutions (NBS) stand out, which respond to ecosystem services and climate challenges, and are classified from the main ecosystems in which they affect: water, vegetation and soil.

Within this context, the interest of the SBN in the international field is analyzed and the adaptation measures included in urban strategies developed to respond to this task are reviewed. The review of interventions in cities of the Mediterranean area makes it possible to value the usefulness of the NBS for urban planning and design.

\section{Key words}

Cities; Climate Change; Adaptation; Nature-Based Solutions; Ecosystems

\section{Resumen}

En los próximos años las ciudades van a sufrir los impactos del cambio climático, que variarán de acuerdo a la configuración topográfica y geométrica urbana, a la distribución y provisión de los espacios verdes, las características de las superficies exteriores y a la densidad demográfica, entre otros aspectos.

Ante esta situación numerosas ciudades han comenzado a adoptar estrategias de adaptación para reducir su vulnerabilidad a los efectos adversos del clima, entre las que destacan las Soluciones Basadas en la Naturaleza (SBN). Estas soluciones favorecen el desempeño de los servicios ecosistémicos en la regulación del clima local, clasificadas según los ámbitos naturales en los que actúan: agua, vegetación y suelo.

A partir de este contexto, se analizan el interés de las SBN en el campo internacional y se revisan las medidas de adaptación incluidas en las estrategias urbanas desarrolladas para dar respuesta a este cometido. La verificación de intervenciones en ciudades del área mediterránea permite poner en valor la utilidad de las SBN para la planificación y el diseño urbano.

Pallabras clave

Ciudades; Cambio Climático; Adaptación; Soluciones basadas en la Naturaleza; Ecosistemas

1 Arquitecta. Universidad San Pablo CEU (Madrid). E-mail: raquelreymelladoarq@gmail.com. ORCID: https://orcid.org/00000001-5167-2809.

2 Paisajista. Doctora en Arquitectura. Profesora de Grado en Paisajismo. Universidad Rey Juan Carlos (Madrid). E-mail: cristina. delpozo@urjc.es. ORCID: https://orcid.org/0000-0001-6217-9921.

3 Paisajista y Arquitecta. Doctora en Arquitectura. Universidad San Pablo CEU (Madrid). E-mail: terefran@temanova.com. ORCID: https://orcid.org/0000-0002-5243-0411. 


\section{Introducción}

El cambio climático está afectando a los ecosistemas urbanos y se prevé que su impacto aumente considerablemente en un futuro próximo. La rápida urbanización ha acelerado la degradación de los ecosistemas urbanos como consecuencia del incremento de las áreas construidas y el consumo de recursos naturales y la pérdida gradual de hábitats naturales. La combinación de los efectos derivados del cambio climático y de la urbanización han dado lugar a numerosos desafíos sociales (Sarabi et al., 2019).

Los impactos de las modificaciones del clima son ampliamente conocidos: aumento de las temperaturas, reducción de las capas de hielo polares, elevación del nivel del mar, alternancia de períodos de sequía y de lluvias torrenciales, incendios forestales, etc. Las implicaciones de estos fenómenos en las ciudades son numerosas, entre ellas, cambios de escorrentías y disponibilidad de agua, desprendimiento de taludes, inundaciones por avenidas, riesgo de erosión y sobrecarga de las infraestructuras de alcantarillado, incremento en el consumo de agua y energía e interrupciones en los suministros, problemas de abastecimiento alimentario e incluso alteración y extinción de especies e incremento de parásitos (Verdaguer Viana-Cárdenas et al., 2015).

Las estrategias de adaptación al cambio climático constituyen una de las principales políticas urbanas dirigidas a mantener la calidad de vida de sus habitantes y a mejorar la resilencia de los tejidos urbanos. La adopción de estas medidas es una actividad ineludible para los gobiernos locales, al ser el nivel de gobierno que asume las competencias de incluir en las estrategias locales las directrices emitidas a nivel europeo, nacional y regional.

Minimizar los resultados de la antropización de los ecosistemas urbanos depende de la localización geográfica de las ciudades y de la capacidad adaptativa de sus estructuras urbanas. En general, estas intervenciones, inciden en la gestión de cuatro aspectos básicos, alentadas por la consecución de objetivos ambientales, económicos y sociales (Oltra y Marín, 2013):

- Gestión de altas temperaturas, a través de medidas reductoras de los episodios de isla de calor en la ciudad, centradas en el tratamiento de los elementos naturales y artificiales constitutivos del espacio urbano.

- Gestión de recursos naturales, especialmente agua y energía, empleando principios bioclimáticos que favorezcan la sustitución de fuentes de energías contaminantes por energías renovables.

- Gestión de catástrofes, elaborando guías de actuación con medidas preventivas adecuadas a las amenazas recurrentes.

- Estrategias indirectas, recurriendo a intervenciones puntuales en el tejido urbano, como es el caso de las soluciones basadas en la naturaleza.

Dentro de estas estrategias, la adopción de criterios de planificación y diseño sostenible que alienten la mezcla de usos, las altas densidades, la reducción de la movilidad motorizada, la regeneración de los tejidos existentes, y la inclusión de principios bioclimáticos que incrementen la biodiversidad, constituyen los principios de acción que incrementan la capacidad de regulación climática de las ciudades (Verdaguer VianaCárdenas et al., 2015). 
En este contexto, las Soluciones Basadas en la Naturaleza (SBN) representan uno de los enfoques más recientes, dirigido a aumentar la resiliencia urbana en el contexto del cambio climático (Kabisch et al., 2016). El aporte de las SBN es relevante en tanto que optan por incluir la naturaleza en la ciudad como medio de atemperación de las condiciones climáticas locales. En definitiva, aprovechan el capital natural para prestar servicios ecosistémicos que cooperan en la resolución de los desafíos del clima.

\section{Marco teórico}

\section{Las soluciones basadas en la naturaleza: definiciones}

Las SBN se refieren a soluciones vivas inspiradas en la naturaleza, que la utilizan y se apoyan en ella para hacer frente a diversos retos ambientales de manera eficiente, a la par de proporcionar simultáneamente beneficios económicos, sociales y medioambientales (Haase $e t$ al., 2017). Dado sus elevados beneficios y co-beneficios potenciales, este tipo de intervenciones se consideran altamente eficientes y rentables para abordar las amenazas del cambio climático (Kabisch et al., 2017). Por estas condiciones pueden ser utilizadas tanto para la gestión de los recursos naturales como para proporcionar servicios ecosistémicos adicionales en beneficio de la salud y el bienestar de los seres humanos y de la propia naturaleza (Eggermont et al., 2015).

El término SBN se utilizó por primera vez a principios de la primera década del siglo XXI, asociado a la interacción entre biodiversidad y bienestar humano. Aun cuando esta relación llevaba algún tiempo siendo reconocida (Berkes, 2012), el término se consolida cuando en 2005 las Naciones Unidas impulsó el proyecto Millenium Ecosystem Assessment (MEA, Evaluación de los Ecosistemas del Milenio) encargado de analizar el estado de los ecosistemas en el planeta. Estos estudios pusieron en evidencia la relación existente entre la degradación global de los ecosistemas y el descenso en la provisión de servicios ecosistémicos, con la consecuente reducción de la calidad de vida de las personas. La MEA puso el énfasis en la necesidad de mejorar la conservación, la restauración y la gestión sostenible de los ecosistemas con el fin de invertir este declive.

En 2013 la Unión Internacional para la Conservación de la Naturaleza (IUCN) definió a las SBN como "acciones para proteger, gestionar y abordar los retos de la sociedad de forma eficaz y adaptativa, proporcionando simultáneamente beneficios para el bienestar humano y la biodiversidad" (Cohen-Shachamet al., 2016). Al operar sobre los ecosistemas, la flexibilidad de las SBN permite su uso en proyectos con distintos objetivos, desde actuaciones de protección y restauración de paisajes, hasta la conformación de infraestructuras verdes, la gestión integrada de zonas especialmente vulnerables o la reducción de riesgos naturales (Cohen-Shachamet al., 2016).

La vigencia de la visión ecosistémica de las SBN fue respaldada en la $13^{\text {a }}$ Conferencia de las Partes del Convenio sobre la Diversidad Biológica celebrada en 2016, donde se reconoció que este tipo de enfoques son "técnicamente factibles, políticamente deseables, socialmente aceptables, económicamente viables y beneficiosos, y que la aplicación y la inversión en estos enfoques están, en general, aumentando a nivel internacional y nacional" (COP del CDB, 2016). A través de estas iniciativas 
internacionales el concepto de SBN fue adoptado y progresivamente incluido en los marcos políticos que buscan "promover las sinergias entre la naturaleza, la sociedad y la economía" (Somarakis et al., 2019).

Según la IUCN, las SBN han de cumplir las siguientes características:

- estar determinadas por los contextos naturales y culturales específicos de cada lugar, incluyendo conocimientos tradicionales, locales y científicos;

- se aplican a una escala de paisaje;

- adoptan normas y principios de conservación de la naturaleza;

- producen beneficios sociales de forma justa y equitativa, de manera que se promueva la transparencia y una amplia participación;

- reconocen y abordan las compensaciones entre la producción de unos pocos beneficios económicos inmediatos para el desarrollo y las opciones futuras para la producción de toda la gama de servicios de los ecosistemas;

- pueden aplicarse individualmente o de forma integrada con otras soluciones a los retos de la sociedad;

- mantienen la diversidad biológica y cultural y la capacidad de los ecosistemas para evolucionar en el tiempo y

- se integran al diseño global de políticas, medidas o acciones destinadas a hacer frente a un reto específico.

En 2020 la IUCN elaboró el Estándar Global que identifica los criterios que deben adoptar las SBN (IUCN, 2020):

- Criterio 1. Identificar el desafío social al que responderán, garantizando la adopción de diseños que satisfagan las necesidades del bienestar humano.

- Criterio 2. Guiar su diseño según consideraciones espaciales, adoptando un enfoque de paisaje.

- Criterios 3,4 y 5. Asegurar el cumplimiento de las tres dimensiones del desarrollo sostenible: sostenibilidad ambiental, equidad social y viabilidad económica.

- Criterio 6. Equilibrar las compensaciones inherentes a las decisiones asociadas a su gestión, incluyendo la conciliación de los intereses en el corto y el largo plazo.

- Criterio 7. Promover un enfoque de gestión adaptativo, mediante el cual el aprendizaje y la acción se complementen entre sí, para mejorar la propuesta de SBN.

- Criterio 8. Promover su integración en la política nacional, fundamental para sustentar su sostenibilidad y durabilidad en el largo plazo, criterio que puede alienarse con las políticas y los compromisos internacionales y el intercambio de lecciones sobre otras soluciones adoptadas.

Estos criterios, además de ofrecer una visión global sobre las $\mathrm{SBN}$, permiten el desarrollo de guías de diseño y de implementación que incrementen la resiliencia de las intervenciones y ofrezcan resultados económicamente viables, ambientalmente racionales y socialmente justos (Sarabi et al., 2019). Sus contenidos han sido progresivamente incorporados en las políticas, estrategias, planes, investigaciones y acciones específicas adoptadas por las distintas naciones para la protección de la biodiversidad y los efectos del cambio climático. 


\section{El interés de la Comisión Europea en las SBN}

En 2015 la Comisión Europea adoptó la definición de la IUCN apoyando el uso de las SBN en tanto que emplean soluciones que excluyen métodos que alteran artificialmente la naturaleza, como los organismos genéticamente modificados (Comisión Europea, 2015).

Según la Comisión Europea, el principal objetivo de las SBN es la adaptación de las ciudades al cambio climático, considerándolas como herramientas de planificación y diseño. Para incentivar el uso de los recursos naturales locales, incrementar la competitividad regional y aplicar soluciones alternativas a las tradicionales formas de intervención urbana y territorial, la Comisión ha generado instrumentos especiales de financiación.

El Programa Horizonte 2020 ha propuesto distintos criterios para enfocar, guiar y evaluar la implementación de un escenario basado en la naturaleza: integrar las $\mathrm{SBN}$ en las agendas internacionales de $\mathrm{I}+\mathrm{D}$; innovar, adaptar, mejorar, evaluar y generar una base de conocimiento de SBN; crear una Comunidad Europea de Innovación e Investigación y mejorar el marco SBN en las políticas de la UE. Además, el programa favorece el desarrollo de proyectos que demuestren la aplicación y sus efectos en el aumento de la producción del bienestar a menores costos y en la oferta de un potencial de innovación.

En 2015 el Grupo de Expertos sobre el Horizonte 2020 de la UE publicó el documento "Soluciones Basadas en la Naturaleza y ciudades renaturalizadas", donde se establece el Programa de Trabajo 2014 para el reto social "Acción por el clima, el medioambiente, la eficiencia de los recursos y las materias primas". En este texto se recogen las SBN que proporcionan beneficios ambientales, sociales y económicos, como consecuencia de acercar la naturaleza y proponer procesos más naturales para las ciudades y los paisajes terrestres y marinos (Comisión Europea, 2015). Asimismo, se identifican los objetivos claves que abordan las SBN: mejorar la urbanización sostenible; estimular el crecimiento económico; mejorar el medioambiente de modo que se consigan ciudades más atractivas y que contribuyan a la mejora del bienestar humano; utilizar la restauración de los ecosistemas degradados mejorando su resiliencia; permitir la adaptación y la mitigación al cambio climático; proporcionar respuestas más resilientes y mejorar la gestión de riesgos y la resiliencia consiguiendo mayores beneficios que los métodos convencionales.

La Comisión Europea promovió durante la Cumbre de Acción Climática de las Naciones Unidas de 2019 el uso de SBN en una amplia gama de áreas políticas, siendo destacable el papel que les concede en el Pacto Verde Europeo aprobado en 2019 y en las políticas de regeneración urbana impulsadas para promover cambios sociales. Según la Estrategia de la UE sobre la Biodiversidad para 2030, adoptada en 2020, las SBN serán esenciales para reducir las emisiones y adaptarse al clima cambiante y deberían "integrarse sistemáticamente en la planificación urbana, incluso en los espacios públicos, las infraestructuras y el diseño de los edificios y su entorno" (Comisión Europea, 2020b). Esta meta está respaldada por los objetivos e iniciativas de la Estrategia, dedicando una proporción significativa del presupuesto a la acción climática, la inversión en biodiversidad y SBN. Con el subtítulo "Devolver la naturaleza a nuestras vidas", la Estrategia da prioridad a "devolver la salud a la naturaleza" y "devolver al planeta más de lo que se le quita", y sugiere que se incremente la implantación y la ampliación de las SBN para que esto sea una realidad (Comisión Europea, 2020a). 
En esta línea, la Comisión Europea promueve las SBN a través de una amplia gama de áreas políticas y apoyó el "Manifiesto SBN para el Clima" propuesto en la Cumbre de Acción Climática de la ONU en 2019. Las SBN se incluyen en la transición europea propuesta por el Pacto Verde - Green Deal- que considera la regeneración urbana como un factor esencial para el cambio social transformador.

En este contexto, la UE financió el proyecto Urban Green Up para el período 2017-2022, destinado a desarrollar, aplicar y validar una metodología para la restauración de planes urbanos con el fin de adaptar las ciudades al cambio climático, mejorar la calidad del aire, gestionar el agua y aumentar la sostenibilidad urbana a través de soluciones innovadoras basadas en la naturaleza. El proyecto elaboró el Catálogo Urban Green Up, donde se materializan los tipos genéricos de SBN en acciones específicas para su incorporación al entorno urbano. El catálogo ofrece ejemplos de aplicación y las correspondientes soluciones técnicas, incluyendo los desafíos a los que se enfrenta su implementación. Entre estos retos destacan la forma construida de la ciudad, el presupuesto municipal disponible, los desafíos que la ciudad desea abordar, el contexto social, cultural, legal y político local y la capacidad de las instituciones que intervienen en el diseño, construcción y mantenimiento de las SBN. Otros programas y proyectos que trabajan con SBN (20072022) son:

- CLEARINH HOUSE: investigación sobre los bosques y árboles urbanos como soluciones basadas en la naturaleza. Se investiga la contribución de los árboles tradicionales urbanos para hacer ciudades sostenibles. El proyecto tiene como objetivo desarrollar una aplicación en línea, una herramienta de referencia global y pautas para respaldar el diseño, gobernanza y ordenación de los bosques urbanos.

- CLEVER Cities: tiene como objetivo aumentar y mejorar el conocimiento local de soluciones basadas en la naturaleza, demostrar que las ciudades más verdes funcionan mejor para las personas y comunidades, contribuir con datos e información a la UE para realizar políticas y promover y permitir la adopción de soluciones basadas en la planificación urbana en todo el mundo.

- CONNECTING Nature: plantea acciones para alimentar la iniciación y expansión de las empresas en la producción e implementación a gran escala de SBN en entornos urbanos para medir el impacto de estas iniciativas en la adaptación al cambio climático, la salud y el bienestar, la cohesión social y el desarrollo económico sostenible.

- EdiCitNet: Red de Ciudades Comestibles se enfoca en soluciones comestibles, definidas como SBN relacionadas con el uso de la producción, distribución y uso urbano de alimentos. Implementa, monitorea y transfiere Edible City Solutions en estrecha cooperación con las autoridades de la ciudad y otras partes interesadas. Por lo tanto, tiene como objetivo aumentar el impacto social y medioambiental, y la sostenibilidad económica de las ciudades.

- GROW GREEN: tiene como objetivo invertir en SBN (espacios verdes de alta calidad y vías fluviales) a través de una planificación urbana a largo plazo para desarrollar el clima y resiliencia hídrica, ciudades fuertes y habitables, capaces de afrontar grandes desafíos urbanos, como inundaciones, estrés por calor, se- 
quía, pobreza, calidad del aire, desempleo y pérdida de biodiversidad.

- Nature4Cities: tiene como objetivo establecer un equilibrio positivo entre los beneficios y costos ambientales y sociales mediante la creación de una plataforma de referencia para las SBN que ofrece soluciones técnicas, métodos y herramientas para la planificación urbana. Este equilibrio implica modelos colaborativos de ciudadanos, investigadores, responsables políticos y líderes de la industria a través de procesos de cocreación.

- NATURVATION: evalúa los logros de las SBN en las ciudades, examina su proceso de innovación y trabaja con las comunidades y las partes interesadas para desarrollar conocimiento y herramientas necesarias para el reconocimiento del potencial de las SBN para alcanzar los objetivos de sostenibilidad urbana.

- REGREEN: tiene como objetivo promover sustancialmente la evidencia y las herramientas al modelar y combinar sistemáticamente los servicios de los ecosistemas y la biodiversidad como base para las SBN urbanas en Europa y China. Esto también implica el aprendizaje experimental de políticas, las estrategias para el desarrollo, la educación y la ciencia ciudadana en las escuelas, la valoración de beneficios y costos y el desarrollo de modelos comerciales para realizar SBN especialmente relevantes que brinden múltiples servicios ecosistémicos y bienestar.

- ThinkNature: desarrolló una plataforma que respalda la amplia comprensión y promoción de las SBN.

- TURaS: muestra ejemplos para mejorar el desarrollo y sostenibilidad urbana, por ejemplo, muros vegetales que se pueden implantar en cualquier lugar con un coste asequible.

- UNaLab: tiene como objetivo desarrollar un marco de referencia europeo sobre beneficios, rentabilidad, viabilidad económica y replicabilidad de las SBN mediante la promoción de comunidades urbanas inteligentes, inclusivas, resilientes y sostenibles a través de la creación conjunta de Urban Living Lab, demostraciones, experimentos y evaluación de SBN para desafíos climáticos e hídricos.

- URBiNAT: se centra en la regeneración e integración de barrios de viviendas sociales desfavorecidos. Las intervenciones se centran en el espacio público para co-crear con los ciudadanos nuevas relaciones urbanas, sociales y basadas en la naturaleza, dentro y entre diferentes barrios. Tiene como objetivo coplanificar un corredor saludable como una SBN innovadora y flexible, integrando las SBN micro que surgen de los procesos de diseño impulsados por la comunidad.

\section{Metodología}

La metodología diseñada para la realización de esta investigación se desarrolló en dos fases:

Durante la primera fase se clasificaron los tipos genéricos de SBN que inciden sobre los distintos ecosistemas, de manera que se pudieran agregar las SBN según sea el componente natural sobre el que actúan: agua, vegetación y suelo.

En la segunda fase se seleccionaron casos de experiencias de implementación SBN en ciudades de clima mediterráneo. Se analizaron in- 
tervenciones de SBN realizadas con el fin de adoptar medidas específicas para atenuar la vulnerabilidad de las ciudades de la zona y clima mediterráneo a los efectos del cambio climático. Las ciudades seleccionadas fueron: Marsella y Montpellier (Francia), Lisboa y Oporto (Portugal), Barcelona (España), Bari (Italia) y Atenas (Grecia).

A partir de la experimentación de las incidencias de las SBN en ciudades del área mediterránea se hizo un análisis comparativo que permitió extraer tanto directrices generales como líneas de acción para ser replicadas en ciudades de características similares.

\section{Fase 1. Clasificación de SBN según componente natural:} agua, vegetación y suelo

\section{Agua}

Las SBN que apoyan a la gestión de recursos hídricos permiten mejorar su uso aplicando dos modalidadesde soluciones tipo:

- Naturalización, conservación y restauración de los cursos de agua: el tratamiento de los elementos de la red hidrográfica —ríos, arroyos, humedales, reservas marinas-influyen tanto en los servicios ecosistémicos como en los objetivos de adaptación climática, además de incrementar su valor paisajístico y ambiental y de generar espacios naturales de ocio y recreo para los ciudadanos.

- Recolección, tratamiento, infiltración y almacenamiento de agua: intervenciones que favorecen la gestión del agua de distintas maneras, por ejemplo, interviniendo en la regulación de los caudales de agua, la incorporación de sistemas de drenaje sostenible, el uso de superficies permeables, o la construcción de estanques de detención y retención y jardines de lluvia.

Ambos tipos de intervenciones contribuyen a la mejora de los servicios ecosistémicos a través del enfriamiento del aire (evapotranspiración), la regulación hídrica (transporte de agua, infiltración de agua, retención de agua), la purificación del agua (filtrado de agua), el mantenimiento de la biodiversidad (provisión de hábitat y conectividad, reintroducción de especies autóctonas, control de especies exóticas invasivas) y los aportes de carácter social (belleza, funcionalidad e interacción social).

Vegetación

La vegetación es, junto a la gestión del agua, uno de los principales recursos para la adaptación de las ciudades a los efectos del clima en tanto que regulan la calidad del aire, reducen las altas temperaturas y controlan las escorrentías. El aumento de la masa vegetal en cualquiera de sus escalas, desde el territorio al edificio, es fundamental para afrontar estos retos. Las SBN que se apoyan en la vegetación se catalogan en cuatro tipos básicos:

- Reforestación o introducción de usos agrícolas: acciones que restauran y conservan los entornos naturales próximos a las áreas urbanas y salvaguardan y mejoran sus cualidades ambientales.

- Corredores vegetales, parques, plazas y jardines urbanos: estas SBN generan redes que utilizan servicios ecosistémicos diversos 
para conectar las ciudades con el entorno natural y articular los tejidos urbanos interiores. Los corredores vegetales reducen la temperatura ambiente en $2^{\circ} \mathrm{C}$, capturan partículas contaminantes, mejoran la calidad del aire, conservan la biodiversidad, aumentan los tipos de especies florales y consolidan la conectividad ecológica. La conservación y restauración de parques, plazas y jardines incrementan sus funciones ecosistémicas, favorecen la biodiversidad e incrementan el interés natural y el uso social de estos espacios.

- Renaturalización y espacios de oportunidad de escala barrial: la inclusión de plazas y espacios ajardinados permite a las áreas densamente pobladas reducir el efecto de isla de calor a la vez que mejoran la calidad de vida de sus habitantes.

- Cubiertas y fachadas vegetales: los objetivos de estas intervenciones son mejorar la calidad del aire, eliminar los contaminantes del aire y dióxido de carbono, reducir la temperatura de aire, aumentar la concentración de oxígeno y restaurar los ecosistemas, además de contribuir a una composición urbanística más amable.

Suelo

Además de absorber y almacenar el exceso de agua y de producir alimentos, el suelo funciona como sumidero de carbono natural al capturar dióxido de carbono del aire. El proyecto CAPRESE- Soil (Lugato et al., 2013) concluyó que la conversión de tierras cultivables en pastos aumenta rápidamente el carbono del suelo. Asimismo, el uso diferente del suelo puede cambiar sus características, convirtiéndolo en fuente de emisiones de carbono previamente almacenado. Este es el caso del drenaje de turberas, la combustión de turba de pantanos, el arado de pastos o el cultivo de las tierras.

Dos son las principales SBN utilizadas para contribuir a la mejora de la calidad de suelo:

- Biochar: se trata de un material de carbón altamente poroso, producido por pirólisis de biomasa que, agregado al suelo favorece el almacenamiento estable de carbono a largo plazo, reduce las emisiones de GEI, mejora la fertilidad, atenúa la declinación de las propiedades químicas, físicas y biológicas, absorbe los contaminantes de la escorrentía urbana de aguas superficiales y libera lentamente los nutrientes de las plantas.

- Agrosilvicultura: sistema que integra árboles, ganado y pastos en la misma unidad productiva, lo que mejora la calidad del suelo y su productividad, reduce las altas temperaturas, aumenta la biodiversidad, controla las plagas y potencia la polinización, reduce la erosión y previene de los daños causados por las inundaciones (Oppla, 2020).

A continuación, se expone un cuadro donde se vincula la relación existente entre las SBN y los servicios ecosistémicos (Cuadro 1): 


\begin{tabular}{|c|c|c|c|c|c|c|c|c|}
\hline \multirow{2}{*}{\multicolumn{2}{|c|}{ SBN }} & \multicolumn{7}{|c|}{ Servicios ecosistémicos } \\
\hline & & \multirow{2}{*}{$\begin{array}{c}\text { SSEE } \\
1 \\
\\
\end{array}$} & \multirow{2}{*}{$\begin{array}{c}\text { SSEE } \\
2 \\
\end{array}$} & \multirow{2}{*}{$\begin{array}{c}\text { SSEE } \\
3 \\
\\
\bullet\end{array}$} & \multirow{2}{*}{$\begin{array}{c}\text { SSEE } \\
4 \\
\\
-\end{array}$} & \multirow{2}{*}{$\begin{array}{c}\text { SSEE } \\
5 \\
\bullet\end{array}$} & \multirow{2}{*}{$\begin{array}{c}\text { SSEE } \\
6 \\
\end{array}$} & \multirow{2}{*}{$\begin{array}{c}\text { SSEE } \\
7 \\
\\
-\end{array}$} \\
\hline \multirow{2}{*}{ Agua } & $\begin{array}{l}\text { Naturalización, conserva- } \\
\text { ción y restauración de los } \\
\text { cursos de agua }\end{array}$ & & & & & & & \\
\hline & $\begin{array}{l}\text { Recolección, tratamiento, } \\
\text { infiltración y almacena- } \\
\text { miento de agua }\end{array}$ & - & $\bullet \bullet$ & • & - & • & • & - \\
\hline \multirow{6}{*}{ Vegetación } & $\begin{array}{l}\text { Reforestación o introduc- } \\
\text { ción de usos agrícolas }\end{array}$ & $\bullet$ & $\bullet$ & - & $\bullet$ & $\bullet \bullet$ & $\bullet$ & $\bullet \bullet$ \\
\hline & Corredores vegetales & $\bullet$ & $\bullet$ & $\bullet$ & $\bullet \bullet$ & $\bullet \bullet$ & $\bullet \bullet$ & - \\
\hline & $\begin{array}{l}\text { Parques, plazas y jardines } \\
\text { urbanos }\end{array}$ & $\bullet$ & $\bullet \bullet$ & • & $\bullet \bullet$ & $\bullet$ & $\bullet$ & - \\
\hline & $\begin{array}{l}\text { Renaturalización y espa- } \\
\text { cios de oportunidad }\end{array}$ & $\bullet$ & • & $\bullet$ & $\bullet \bullet$ & $\bullet \bullet$ & $\bullet \bullet$ & $\bullet \bullet$ \\
\hline & Cubiertas vegetales & $\bullet \bullet$ & $\bullet \bullet$ & $\bullet$ & • & • & $\bullet$ & $\bullet \bullet$ \\
\hline & Fachadas vegetales & $\bullet$ & - & - & • & $\bullet$ & $\bullet$ & - \\
\hline Suelo & Biochar/Agrosilvicultura & $\bullet$ & $\bullet$ & $\bullet$ & $\bullet \bullet$ & $\bullet$ & - & $\bullet \bullet$ \\
\hline
\end{tabular}

Cuadro 1: Vinculación SBN y SSEE. Fuente: elaboración propia.

\section{Fase 2. Intervenciones de SBN en ciudades mediterráneas}

El estudio de casos es útil para examinar en profundidad fenómenos y relaciones sociales complejas con referencia a sus contextos reales (Yin, 2009). El conocimiento adquirido de experiencias a través de proyectos es fundamental para reforzar el empleo de SBN en la planificación y el diseño urbanos (Kabisch et al., 2016).

En la segunda fase, se analizan e investigan un conjunto de catorce intervenciones de SBN, emprendidas en ciudades de clima y área mediterránea, que contribuyen directamente a la adaptación al cambio climático. La experimentación de las intervenciones de SBN permite detectar los aspectos sustantivos que sostienen las propuestas para adaptar las ciudades a los efectos del cambio climático, y sistematizar y extraer unas directrices generales a modo de conclusiones que podrán ser replicadas en la planificación urbana de ciudades de características similares.

En el análisis de las intervenciones, clasificadas a partir de los componentes del ecosistema (fase 1), se han estudiado las siguientes variables: las características de cada una de las SBN empleadas, los desafíos a los que se enfrenta, su contribución ecosistémica a la adaptación de las ciudades al cambio climático, las instituciones involucradas, los procesos participativos, las respuestas a las diferentes estrategias y políticas europeas, nacionales y locales y la realización de evaluaciones mediante monitoreo de las acciones (Cuadro 2) (Naturvation, 2017, 2018; Oppla, 2018, 2020).

A continuación, se exponen diferentes intervenciones a partir de la clasificación propuesta: 


\section{Agua}

Naturalización, conservación y restauración de los cursos de agua

La intervención en el río Aygalades en Marsella (Francia) (Naturvation, 2018a), sobre el componente ecosistémico "agua", consistió en la reconversión del río en un corredor fluvial a través de la incorporación de la vegetación autóctona, integración de espacios libres e instalación de una cuenca de filtración natural.

Estas actuaciones contribuyeron tanto a la mejora ecosistémica como a la adaptación climática. Los principales agentes intervinientes en este proyecto fueron la Agencia de Planificación Euroméditerranée con el apoyo de la UE, los gobiernos regional y municipal y la participación ciudadana. Asimismo, se comprueba que el proyecto responde a la estrategia nacional "Etiqueta Eco-citê" del Ministerio de Ecología francés.

\section{Recolección, tratamiento, infiltración y almacenamiento de agua}

"Las cinco nuevas cuencas de retención de aguas pluviales" en Marsella (Francia) (Naturvation, 2017a) se trata de una intervención que actuó sobre el componente ecosistémico "agua" a través de la recolección, tratamiento, infiltración y almacenamiento. Tras comprobar que el sistema de redes de alcantarillado existente se desbordaba ante tormentas severas y descargaba el agua en el mar sin tratamiento alguno, se construyeron cinco cuencas de retención para mejorar de forma directa la regulación hídrica, purificación de agua y aire y de forma indirecta aumentar la biodiversidad, conservar la vida acuática y mejorar el carácter social.

Este proyecto, organizado de forma conjunta por los gobiernos nacional, regional y local y el sector privado, respondió a estrategias europeas y nacionales sobre "Agua y medio marino"y "Aguas residuales urbanas", y a estrategias locales en relación al tratamiento de las aguas residuales de la ciudad y la calidad de las aguas costeras y los ríos urbanos.

Ante un reto similar se ejecuta el "sistema de drenaje sostenible del Parque Joan Reventós” (Barcelona, España) (Naturvation, 2018b), situado dentro de la zona de captación de agua "Riera de les Monges". La intervención tenía como objetivos absorber, filtrar, reutilizar el agua, evitar inundaciones, incrementar áreas de recreo y conectar vegetalmente áreas urbanas. Estos objetivos se consiguieron mediante la instalación de un sistema de drenaje sostenible, la conservación de la vegetación existente y la incorporación de nuevas plantaciones procedentes de la Sierra de Collserola.

El gobierno municipal de Barcelona ejecutó este proyecto haciendo partícipe a la ciudadanía y sin dar respuesta a estrategias o políticas específicas.

\section{Vegetación}

\section{Reforestación o introducción de usos agrícolas}

Las intervenciones realizadas en el "Parque Collserola" (Oppla, 2018a), situado al norte de la ciudad de Barcelona (España), estaban enfocadas a la protección del hábitat natural y educación ambiental con el fin de preservar los valores naturales y promover el uso sostenible y respetuoso del parque. Estas actuaciones, estudiadas por Depietri et al. (2016), contribuyeron principalmente a mejorar la habitabilidad, 
reducir las temperaturas y eliminar la contaminación en la ciudad de Barcelona.

El gobierno municipal, a través del Consorcio del Parque Natural de la Serra de Collserola, desarrolló el Plan Especial de Ordenamiento y Protección del Medio Natural del Parc de Collserola, y hace partícipe a los ciudadanos a través de la difusión de información, actividades educativas, etc.

Además, este proyecto responde a la Directiva 92/43/CEE de 21 de mayo de 1992 sobre la conservación de hábitats naturales, fauna y flora silvestres y a la estrategia local en el marco del Plan de Protección especial que incluye el Plan Especial de Protección y Medio Ambiente del Parc del Collserolla, el Plan de Espacios de Interés Natural y el Plan Especial de Ordenación y Protección del Parque Natural de Collserola.

Otro proyecto de características similares es "100000 árboles en el área de Oporto" (Portugal) (Naturvation, 2018c), a partir del cual diferentes agentes (propietarios de tierras, gobierno municipal, voluntarios, ONGs, etc.) plantaron árboles autóctonos en 40 hectáreas de propiedad pública y privada. Los principales objetivos de estas actuaciones son reforestar la zona con especies autóctonas, crear oportunidades de formación e impactar sobre la conciencia y el conocimiento de las personas, estructurar programas de voluntariado para la reforestación, promover el cambio social y la participación ciudadana y contribuir a los servicios ecosistémicos.

Este proyecto lo organizó el Centro Regional de Excelencia en Educación para el Desarrollo Sostenible del Área Metropolitana de Oporto, coordinado por el Área Metropolitana de Oporto y la Universidad Católica Portuguesa, y dio respuesta al Plan Estratégico de Ambiente del Área Metropolitana de Oporto (2003-2008). La ejecución de este proyecto permitió comprobar que la plantación de 100000 árboles contribuyó al secuestro de 20280 toneladas de carbono en esta zona.

Corredores vegetales, parques, plazas y jardines urbanos

A una escala más urbana se identifica la intervención "Corredor Verde Sant Joan” (Barcelona, España) (Naturvation, 2018d), que se ejecutó con el fin de incrementar la conectividad ecológica y social de la ciudad. El corredor conecta el Parque de Sant Joan con el Parque de la Ciutadella e incorpora espacios para peatones y niños, pavimentos permeables y sistemas de arbustos y árboles autóctonos. A través de estas actuaciones se contribuyó a mejorar el carácter social, la biodiversidad y sostenibilidad.

El Ayuntamiento de Barcelona promovió este proyecto para dar respuesta al Plan de Infraestructura Verde y Biodiversidad de Barcelona 2020, la Estrategia de Biodiversidad 2020 de la UE y la estrategia local del Plan de Infraestructura Verde y Biodiversidad de Barcelona 2020. El gobierno municipal hizo partícipe a la ciudadanía en este proyecto a través de la difusión de información, actividades educativas y supervisión ciudadana a través de juntas, asesorías, etc.

Otra actuación en esta línea de intervención es el "Corredor Verde de Monsanto" (Lisboa, Portugal) (Oppla, 2018b), que conecta el centro de la ciudad con el parque de Monsanto a través de un sistema ecológico continuo que combina movilidad, circulación de agua y aire y transición fluvial. Los objetivos de estas actuaciones eran mejorar la calidad de vida en la ciudad, resolviendo los desafíos urbanos como el efecto isla de calor, inundacionesy contaminación del aire; potenciar el 
atractivo de la ciudad mediante espacios de recreo; incrementar el valor de la propiedad y mejorar las conexiones y los ecosistemas.

El proyecto fue desarrollado por el gobierno municipal dando repuesta a la estrategia del Plan Maestro de Desarrollo de Lisboa aprobado en 2012, a la Estrategia Municipal de Adaptación al cambio climático de Lisboa 2016 y a la Estrategia Biodiversidad 2020.

Tras su ejecución se comprueba que se han proporcionado 900 hectáreas de área verde a los ciudadanos, que mejoran las conexiones ecológicas, contribuyen al control de la contaminación atmosférica, generan una ciudad más atractiva y conectan los espacios verdes.

\section{Renaturalización y espacios de oportunidad}

A una escala más pequeña se desarrollan proyectos vegetales como "Calles vegetales" en Marsella (Francia) (Naturvation, 2017b). Este proyecto nació con la implementación de los permisos "Visa Verde" y "Carta para la vegetación de espacios públicos de Marsella", dados a la ciudadanía para ocupar el espacio público mediante la instalación de plantas, flores, arbustos y contribuir a hacer una ciudad más atractiva, fomentar la biodiversidad, reducir la contaminación del aire, regular la temperatura, mitigar los efectos de la impermeabilización del suelo y crear vínculos sociales.

Este proyecto fue llevado a cabo por el gobierno local y organizaciones no gubernamentales para dar respuesta al Plan Territorial de Clima.

El objetivo de enverdecer los espacios públicos también se ve reflejado en el "Jardín de los sentidos" en Bari(Italia) (Naturvation, 2017c). Tenía como fin repensar los espacios urbanos para reconvertirlos en áreas verdes y jardines temáticos haciendo partícipe a la ciudadanía. Estas intervenciones contribuyeron a mejorar servicios ecosistémicos como la biodiversidad, el enfriamiento y el carácter social.

Este proyecto, promovido por el gobierno municipal, no responde a ningún tipo de estrategia o política europea, nacional y municipal.

Otras acciones renaturalizan los espacios urbanos abandonados como los "Huertos en Marousi" en Atenas (Grecia) (Naturvation, 2017d). Este proyecto es un ejemplo de reestructuración del espacio público abandonado en huertos privados de hortalizas y frutas. Esta acción contribuyó a mejorar servicios ecosistémicos de provisión, regulación hídrica, purificación del aire, biodiversidad y carácter social.

Además, ha sido llevado a cabo por la labor colaborativa entre el gobierno y la ciudadanía y responde a la directiva alimentaria Europea mediante la donación al banco de alimentos del $20 \%$ de la producción.

En esta línea de intervención se ejecutó también el proyecto "Huertos urbanos del Poblenou” (Barcelona, España) (Naturvation, 2017e), que convirtió un solar ocupado por restos de un edificio demolido en un jardín para el entretenimiento social e intercambio de conocimiento sobre agricultura. Este proyecto se promovió por los ciudadanos y grupos comunitarios dirigidos por actores no gubernamentales, se ejecutó sin responder a estrategias o políticas europeas, nacionales o locales y contribuyó a mejorar los servicios de provisión, regulación hídrica, purificación del aire, biodiversidad y carácter social.

\section{Cubiertas y fachadas vegetales}

Algunos ejemplos de proyectos analizados correspondientes a la clasificación de vegetación en cubiertas y fachadas vegetales son la cubier- 
ta ajardinada de la Escuela Ferrán Suyner (Barcelona, España) y el edificio "Nueva Naturaleza" (Marsella, Francia).

La cubierta ajardinada de la Escuela Ferrán Suyner (Naturvation, 2017f) permitió al alumnado adquirir conocimientos sobre horticultura y valores sobre los alimentos, contactar con la naturaleza, cuidar el medio ambiente y la ecología, reciclar materiales y recolectar vegetales orgánicos. Estas acciones contribuyeron a mejorar los servicios de provisión, regulación hídrica, y carácter social.

Por otro lado, el edificio "Nueva Naturaleza" (Naturvation 2018e), diseñado por Jean Nouvel, utiliza la naturaleza de forma omnipresente desde el suelo hasta las fachadas y las cubiertas del edificio. Las fachadas incorporan árboles y las cubiertas jardines comunitarios que incorporan espacios para la horticultura y encuentros vecinales, contribuyendo así a reducir la contaminación del aire y acústica, mejorar el vínculo social y el consumo saludable.

\section{Suelo}

\section{Biochar y agrosilvicultura}

Por último, se han identificado pocos proyectos relacionados con el ecosistema "suelo", biochar o agrosilvicultura. Se destaca el sistema agrícola utilizado en Montpellier (Francia) (Oppla, 2020) en el que se combina la plantación de nogales y el cultivo de trigo. Este sistema aumenta la resiliencia a los efectos climáticos, la producción agrícola y biodiversidad, la conectividad ecológica, la purificación del aire y la infiltración y almacenamiento de agua. Estas acciones son promovidas desde el sector agrícola.

A partir del análisis de estas intervenciones se identifican una serie de parámetros SBN que permiten comparar cualitativamente los diferentes proyectos de ciudades mediterráneas y obtener unas directrices de aplicación de soluciones naturales en ciudades de características similares (Cuadro 2).

\begin{tabular}{|c|c|c|c|c|c|c|c|c|c|c|c|c|c|c|c|c|c|c|c|c|}
\hline \multirow[b]{3}{*}{ Proyectos } & \multicolumn{20}{|c|}{ Parámetros SBN } \\
\hline & \multicolumn{5}{|c|}{$\begin{array}{l}\stackrel{0}{0} \\
\stackrel{0}{\tilde{n}} \\
\stackrel{0}{0}\end{array}$} & \multicolumn{7}{|c|}{ 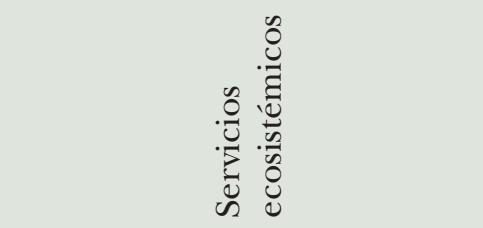 } & \multicolumn{2}{|c|}{ 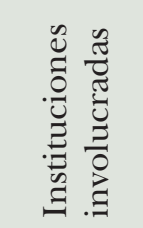 } & \multirow[t]{2}{*}{ 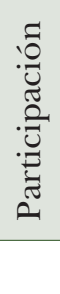 } & \multicolumn{3}{|c|}{ 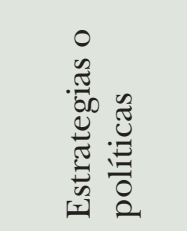 } & \multicolumn{2}{|c|}{ 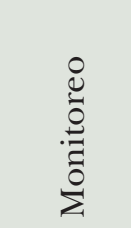 } \\
\hline & $\mathrm{a}$ & $\mathrm{b}$ & c & $\mathrm{d}$ & $\mathrm{e}$ & 1 & 2 & 3 & 4 & 5 & 6 & 7 & $\mathrm{~PB}$ & PV & & $\mathrm{E}$ & $\mathrm{N}$ & $\mathrm{L}$ & SI & $\mathrm{NO}$ \\
\hline Río Aygalades & $\bullet$ & $\bullet$ & & $\bullet$ & & $\bullet$ & $\bullet$ & & - & $\bullet$ & - & & $\bullet$ & & - & & $\bullet$ & & $\bullet$ & \\
\hline $\begin{array}{l}\text { Cinco cuencas } \\
\text { retención }\end{array}$ & $\bullet$ & $\bullet$ & & & & & $\bullet$ & $\bullet$ & • & $\bullet$ & $\bullet$ & & $\bullet$ & $\bullet$ & & $\bullet$ & $\bullet$ & & & $\bullet$ \\
\hline $\begin{array}{l}\text { Parque Joan } \\
\text { Reventós }\end{array}$ & $\bullet$ & $\bullet$ & & & & & $\bullet$ & - & $\bullet$ & & $\bullet$ & & $\bullet$ & & $\bullet$ & & & & & $\bullet$ \\
\hline Parque Collserola & & & & $\bullet$ & & $\bullet$ & & & & - & $\bullet$ & & $\bullet$ & & $\bullet$ & $\bullet$ & & $\bullet$ & $\bullet$ & \\
\hline 100000 árboles & & & & & & - & $\bullet$ & & 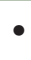 & - & $\bullet$ & & - & & $\bullet$ & & & - & $\bullet$ & \\
\hline Corredor Sant Joan & $\bullet$ & & & • & & & $\bullet$ & & & $\bullet$ & $\bullet$ & & • & & $\bullet$ & - & & $\bullet$ & & - \\
\hline Corredor Monsanto & $\bullet$ & & & - & & - & $\bullet$ & & $\bullet$ & & $\bullet$ & & $\bullet$ & & & & & - & $\bullet$ & \\
\hline Calles vegetales & $\bullet$ & & & $\bullet$ & & $\bullet$ & $\bullet$ & & $\bullet$ & $\bullet$ & $\bullet$ & & • & & - & & & $\bullet$ & & • \\
\hline
\end{tabular}




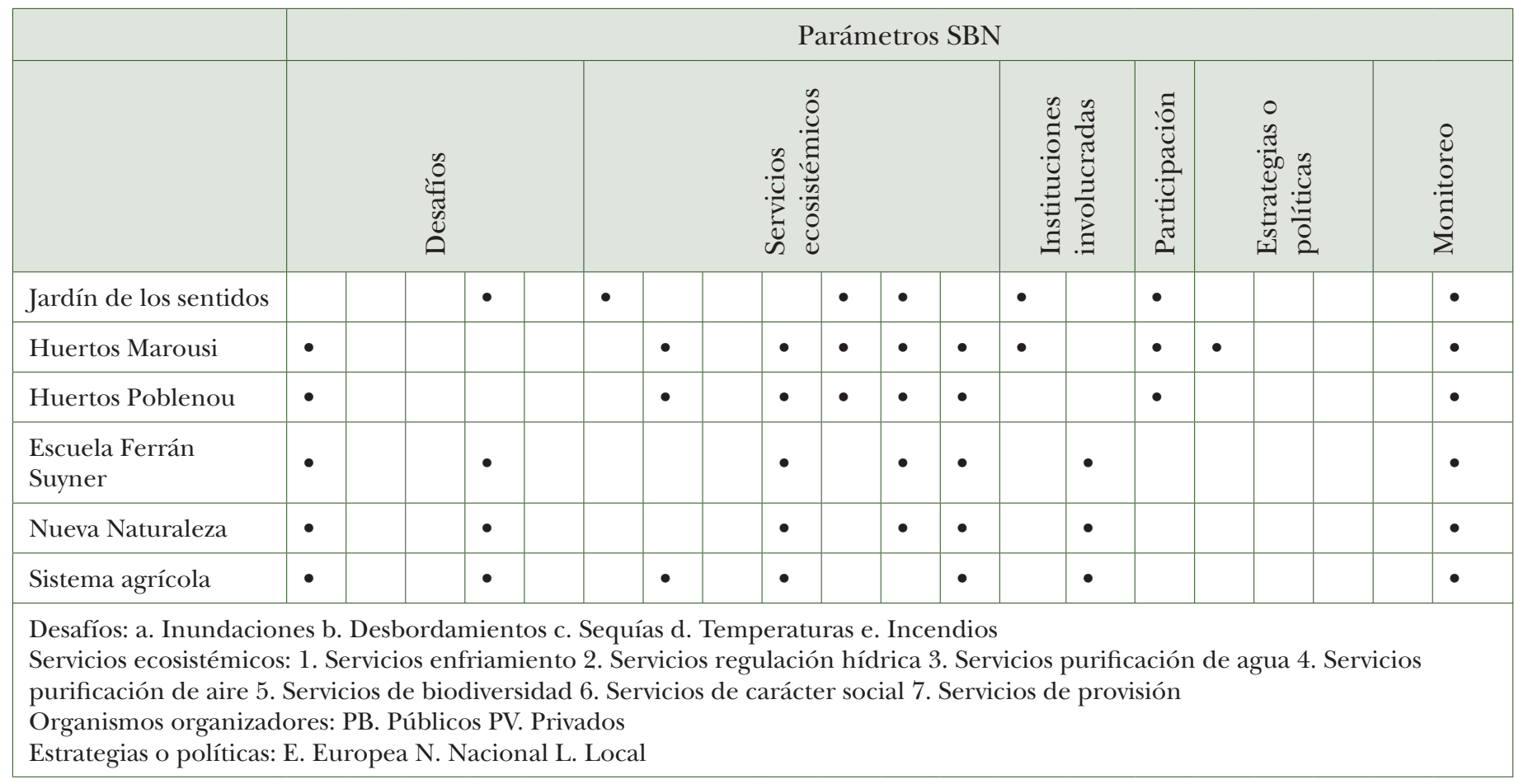

\section{Resultados}

Del análisis comparativo de las intervenciones correspondientes a los casos de ciudades mediterráneasse extraen las siguientes directrices generales:

a) Las $\mathrm{SBN}$, independientemente de la escala, contribuyen a mejorar los servicios ecosistémicos y como consecuencia contribuyen a la adaptación de las ciudades al cambio climático.

b) Los proyectos analizados se desarrollan a distintas escalas: tanto a escala territorial, como en el proyecto sobre el río Aygalades, como a escala urbana en "Calles vegetales" y a escala de edificio como en la "Nueva Naturaleza". Independientemente de la escala, se comprueba que los distintos proyectos contribuyen a mejorar los servicios ecosistémicos y mejoran la adaptación de las ciudades al cambio climático.

c) El efecto acumulativo de las acciones $\mathrm{SBN}$ permite abordar los diferentes desafíos climáticos, pero esta contribución sería mayor si respondieran a un plan de acción urbana que definiera intervenciones de manera multiescalar, desde la visión territorial hasta la escala de barrio y de edificación.

d) Las SBN dan respuesta a los desafíos climáticos urbanos propios de las condiciones de cada lugar. Algunos de los proyectos nacieron para abordar directamente desafíos climáticos, inundaciones y altas temperaturas, como el proyecto "cinco nuevas cuencas de retención de aguas pluviales", sin embargo, otros nacen con un carácter más social como el "Jardín de los sentidos". Ante las diferentes formas de abordar un proyecto, la creación de SBN contribuye de forma directa o indirecta a la adaptación climática.

e) La participación de los gobiernos nacionales y locales, organizaciones, comunidades, asociaciones locales y el sector privado es crucial para la implementación de las SBN. Se comprueba que la mayoría de los proyectos se promovieron desde los gobiernos
Cuadro 2: Análisis comparativo intervenciones SBN. Fuente: elaboración propia. 
municipales dando respuesta a estrategias o políticas locales. Además, se observan diferencias entre los proyectos a escala territorial, como "cinco nuevas cuencas de retención de aguas pluviales" y "Parque de Collserola", que dan respuesta a estrategias europeas y locales en materia de conservación natural y los proyectos de escala de edificación, promovidos por el sector privado y sin responder a ningún tipo de estrategia.

f) Se deben repensar los espacios urbanos existentes y actualizar su infraestructura a través de la renaturalización de calles, espacios abandonados, etc. Las ciudades necesitan ser planificadas desde el punto de vista ecosistémico e incorporar SBN vegetales en espacios urbanos (calles, plazas, jardines, espacios abandonados, etc.) como el "Jardín de los sentidos" o los "Huertos de Marousi" y en edificación como en el edificio "Nueva Naturaleza".

g) Las SBN requieren de un enfoque colaborativo de la gobernanza donde la participación ciudadana sea activa y se involucre en los procesos y programas que conectan con la planificación de SBN a nivel municipal. La mayoría de las intervenciones SBN se desarrollan haciendo partícipe a la ciudadanía en las fases de proyecto, como en el proyecto del "Corredor de Joan Reventós", o de ejecución, como en los "Huertos urbanos del Poblenou".

h) El diseño de SBN debe ser realizado por grupos multidisciplinares profesionales para dar respuesta a los diferentes servicios ecosistémicos y contribuir de forma más eficiente a la adaptación climática. Las diferentes tipologías de SBN existentes que responden a los diferentes servicios ecosistémicos, como la reconversión del río Aygalades (que contribuye a mejorar servicios ecosistémicos de enfriamiento, biodiversidad, purificación del aire, regulación hídrica y carácter social), o el "Jardín Urbano de Ploblenou” (que contribuye a mejorar el sistema de provisión y el carácter social), han de ser diseñadas por grupos multidisciplinares para dar respuestas más eficientes a la adaptación climática.

i) Monitorear la implementación de las SBN para evaluar técnicamente su eficiencia con respecto a las soluciones convencionales y poder mejorar las propuestas de SBN. Los proyectos "Corredor fluvial río Aygalades", "Parque Collserola", "100000 árboles en el área de Oporto" y "Corredor Verde de Monsanto" monitorean su efectividad con el fin de mejorar las soluciones adoptadas. Por ello es recomendable monitorear la implementación de las SBN de manera que se pueda evaluar la efectividad real y temporal de las intervenciones.

$\mathrm{El}$ análisis de las intervenciones $\mathrm{SBN}$ ha permitido extraer siete directrices generales que retroalimentan a los diferentes proyectos. A continuación, se expone el Cuadro 3 en el que se relacionan las directrices generales con las intervenciones: 


\begin{tabular}{|c|c|c|c|c|c|c|c|c|}
\hline \multirow{3}{*}{ Proyectos } & \multicolumn{8}{|c|}{ Directrices generales } \\
\hline & \multirow{2}{*}{ A } & \multicolumn{2}{|c|}{ B } & \multirow{2}{*}{ G } & \multirow{2}{*}{$\mathrm{D}$} & \multirow{2}{*}{$\mathrm{E}$} & \multirow{2}{*}{$\mathrm{F}$} & \multirow{2}{*}{ G } \\
\hline & & Directas & Indirectas & & & & & \\
\hline Río Aygalades & $\bullet$ & - & & - & & - & & $\bullet$ \\
\hline $\begin{array}{l}\text { Cinco cuencas reten- } \\
\text { ción }\end{array}$ & $\bullet$ & • & & $\bullet$ & & & & \\
\hline Parque Joan Reventós & $\bullet$ & $\bullet$ & & $\bullet$ & & $\bullet$ & & \\
\hline Parque Collserola & $\bullet$ & & $\bullet$ & $\bullet$ & & $\bullet$ & & $\bullet$ \\
\hline 100000 árboles & $\bullet$ & & $\bullet$ & $\bullet$ & & - & & $\bullet$ \\
\hline Corredor Sant Joan & $\bullet$ & & $\bullet$ & $\bullet$ & $\bullet$ & $\bullet$ & & \\
\hline Corredor Monsanto & $\bullet$ & $\bullet$ & & - & $\bullet$ & & & • \\
\hline Calles vegetales & $\bullet$ & $\bullet$ & & $\bullet$ & $\bullet$ & $\bullet$ & & \\
\hline Jardín de los sentidos & $\bullet$ & & $\bullet$ & $\bullet$ & $\bullet$ & $\bullet$ & & \\
\hline Huertos Marousi & $\bullet$ & & $\bullet$ & $\bullet$ & $\bullet$ & $\bullet$ & & \\
\hline Huertos Poblenou & $\bullet$ & & $\bullet$ & $\bullet$ & $\bullet$ & $\bullet$ & & \\
\hline Escuela Ferrán Suyner & $\bullet$ & & $\bullet$ & $\bullet$ & $\bullet$ & & & \\
\hline Nueva Naturaleza & $\bullet$ & & $\bullet$ & $\bullet$ & $\bullet$ & & & \\
\hline Sistema agrícola & $\bullet$ & - & & $\bullet$ & & & & \\
\hline
\end{tabular}

Además de las siete directrices generales, las diferentes intervenciones permiten proponer las siguientes estrategias de planificación para la adaptación al cambio climático:

1) Crear campañas de reforestación de zonas degradadas situadas en los entornos naturales de la ciudad para mitigar los efectos del cambio climático y convertir áreas próximas municipales en espacios productivos;

2) Crear corredores ecológicos que comuniquen las zonas verdes existentes (parques, jardines botánicos, plazas, etc.) y los espacios naturales del entorno urbano;

3) Incorporar alrededor de los corredores urbanos y parques próximos espacios multifuncionales que alberguen redes de itinerarios peatonales y ciclistas, áreas de recreo, etc.;

4) Fomentar la infiltración natural de las aguas pluviales minimizando la impermeabilización del suelo para reducir los efectos derivados de las crecidas y lluvias torrenciales y facilitar el cierre del ciclo del agua, creando condiciones adecuadas para la biodiversidad urbana y contribuyendo así a la mitigación mediante la reducción de la artificialización del suelo;

5) Diseñar, integrar y ampliar la red de espacios verdes utilizando vegetación autóctona adaptada y de bajo requerimiento hídrico, la permeabilización del suelo e instalación de masas de agua;

6) Recuperar los espacios infrautilizados o abandonados en las áreas urbanas para generar espacios públicos, huertas o jardines comunitariosy así mejorar la calidad del suelo existente y potenciar las actividades educativas y las interacciones sociales;

7) Generar redes escolares de huertos urbanos ecológicos y
Cuadro 3: Relación entre las directrices generales y proyectos SBN. Fuente: elaboración propia. 
8) Fomentar el uso de sistemas de riego eficaces (reutilización de agua, riego por goteo, etc.).

\section{Conclusiones}

La capacidad de las SBN para adaptar las ciudades al cambio climático se puede comprender a partir de la aproximación de las soluciones adoptadas en los proyectos o intervenciones realizadas en los casos seleccionados.

A partir de este análisis, ha sido posible identificar la existencia de SBN tanto a escala territorial como a escala local. Esto nos permite concluir que las intervenciones a través de SBN han de estar integradas en los planes territoriales y llevar a cabo una planificación multiescalar, desde el territorio hasta la escala de barrio urbano. Además, se ha relacionado cada intervención con sus efectos ante el cambio climático, principalmente: lluvias torrenciales, inundaciones y altas temperaturas.

Por otro lado, la metodología de investigación utilizada ha permitido identificar conclusiones relevantes que se han traducido en directrices y medidas estratégicas de intervención para aplicar en las ciudades mediterráneas y así afrontar los retos climáticos. Los parámetros identificados valoran de forma cualitativa la contribución de las SBN al entorno urbano, lo que permite dirigir la investigación hacia la utilización de las SBN como instrumento de planificación para la adaptación de las ciudades al cambio climático.

Además, es necesario el paso del tiempo para comprobar de forma exhaustiva la evolución de las soluciones adoptadas y su contribución a la reducción del efecto climático, a través de su monitoreo para obtener la cuantificación de las soluciones más efectivas para alcanzar las condiciones de equilibrio climático. Esta evaluación se propone para futuras investigaciones, de modo que, a partir de parámetros medibles exista la posibilidad de calcular de forma óptima las acciones para incorporarlas en los proyectos.

Las SBN conforman ámbitos de innovación en el tratamiento de las ciudades para adaptarlas y hacerlas más resilientes, por tanto, su incorporación en las herramientas de planificación y diseño aportan nuevas visiones y contenidos en la forma de entender y actuar en la red urbana.

Comprender cómo funcionan los procesos ecosistémicos en las ciudades permite aproximar soluciones urbanas para reducir los efectos del cambio climático aprovechando las potencialidades de sus componentes ambientales. Los profesionales del espacio físico y las administraciones públicas que impulsan este tipo de estrategias han de anteponer el valor de la naturaleza ante otro tipo de propuesta urbanística. 


\section{Referencias bibliográficas}

Berkes, Flikret (2012). Implementing ecosystem-based management: evolution or revolution? Fish and Fisheries, 13(4), 465-476. DOI: https://doi. org/10.1111/j.1467-2979.2011.00452.x.

CBD COP (2016, 10 de diciembre). Decisión XIII/4. Biodiversity and climate change. $\mathrm{CBD} / \mathrm{COP} / \mathrm{DEC} /$ XIII/4) Recuperadoel 25 de junio de 2020 de: https://www.cbd.int/meetings/COP-13.

Cohen-Shacham, Emmanuelle, Walters, Gretchen, Janzen, Christiney Maginnis, Stewart (2016). Nature-based Solutions to address global societal challenges. Gland: IUCN. DOI: https:/ / doi.org/10.2305/IUCN. CH.2016.13.en.

Comisión Europea (2015). Towards an EU Research and Innovation policy agenda for Nature-Based Solutions E Re-Naturing Cities. Final ReportoftheHorizon, 2020. Luxemburgo: Oficina de Publicaciones Unión Europea. Recuperado el 25 de junio de 2020 de: https://op.europa.eu/en/publication-detail/-/publication/fb117980-d5aa-46df-8edc-af367cddc202.

Comisión Europea (2020a). A European Green Deal. 6 Commission priorities for 2019-24. Recuperado el 25 de junio de 2020 de: https:/ / ec.europa.eu/info/ strategy/priorities-2019-2024/european-greendeal_en.

Comisión Europea (2020b). Communication from the Commission to the European Parliament, The Council, The European Economic and Social Committee and The Committee of the Regions. EU BiodiversityStrategy for 2030 [COM(2020) 380 final]. Recuperado el 25 de junio de 2020 de:https:/ / eur-lex.europa.eu/resource.html?uri=cellar:a3c806a6-9ab3-11ea-9d2d01aa75ed71a1.0001.02/DOC_1\&format=PDF.

Depietri, Yaella, Kallis, Giorgos, Baró, Francesc y Cattaneo, Claudio(2016). The urban political ecology of ecosystem services: The case of Barcelona. Ecological Economics, 125, 83-100. DOI: https:/ / doi. org/10.1016/j.ecolecon.2016.03.003.

Eggermont, Hilde, Balian, Estelle, Azevedo, José Manuel N., Beumer, Victor, Brodin, Tomas; Claudet, Joachim, ... y X. Le Roux (2015). Nature-based solutions: new influence for environmental management and research in Europe. GAIA Ecological Perspectives, 24 (4), 243-248. DOI: https://doi. org/10.14512/gaia.24.4.9.

Haase, Dagmar, Kabisch, Sigrun, Haase, Annegret, Andersson, Erik, Banzhaf, Ellen, Baró, Francesc, ... y Wolff, Manuel (2017). Greening cities-To be socially inclusive? About the alleged paradox of society and ecology in cities. Habitat International, 64, 41-48. DOI: https://doi.org/10.1016/j.habitatint.2017.04.005.
IUCN (2020). Guidance for using the IUCN Global Standard for Nature-based Solutions. A user-friendly framework for the verification, design and scaling up of $\mathrm{Na}$ ture-based Solutions. Gland: IUCN. DOI: https://doi. org/10.2305/IUCN.CH.2020.09.en.

Kabisch, Nadja, Frantzeskaki, Niki, Pauleit, Stephan, Naumann, Sandra, Davis, McKenna, Artmann, Martina, ... y Bonn, Aletta (2016). Nature-based Solutions to climate change mitigation and adaptation in urban areas: perspectives on indicators, knowledge gaps, barriers, and opportunities for action. Ecology and Society 21(2), 39. DOI: http:/ / dx.doi. org/10.5751/ES-08373-210239.

Kabisch, Nadja, Korn, Horst, Stadler, Jutta y Bonn, Aletta (2017). Nature-based solutions to climate change in urban areas: linkages between science, policy and practice. Cham: Springer Nature. Recuperado el 30 de junio de 2020 de: https://link.springer.com/ book/10.1007/978-3-319-56091-5.

LugatoEmanuele, Bampa Francesca, Montanarella Luca, AngileriVincenzo, Bertaglia Marco, CeglarAndrej, ... y Paloma Sergio (2013).Options and implications for agricultural production-Report of Task 7: Final Report(EUR 26516).Bruxelas: European Union. JRC88295. Recuperado el 30 de junio de 2020 de: https://publications.jrc.ec.europa.eu/repository/handle/JRC88295.

Millenium Ecosystem Assessment (2005). Ecosystems and human well-being: wetlands and water.

Naturvation (2017a). 5 nuevas cuencas de retención de aguas pluviales. Urban Nature Atlas. Recuperado el 5 de junio de 2020 de: https:/ / naturvation.eu/ nbs/marseille/5-new-stormwater-retention-basins.

Naturvation (2017b). Calles vegetales. Urban Nature Atlas. Recuperado el 5 de junio de 2020 de: https:/ / naturvation.eu/nbs/marseille/vegetation-streets.

Naturvation (2017c). Jardín de los sentidos. Urban $\mathrm{Na}$ ture Atlas. Recuperado el 5 de junio de 2020 de: https://naturvation.eu/nbs/bari/garden-senses.

Naturvation (2017d). Jardinería privada de hortalizas y frutas en Marousi. Urban Nature Atlas. Recuperado el 5 de junio de 2020 de: https:/ / naturvation.eu/ nbs/athens / private-vegetable-and-fruit-gardeningmarousi.

Naturvation (2017e). Jardines urbanos de Poblenou. Urban Nature Atlas. Recuperado el 5 de junio de 2020 de: https://naturvation.eu/nbs/barcelona/ urban-gardens-poblenou.

Naturvation (2017f). Jardín en la azotea (Escuela Ferrán Sunyer). Urban Nature Atlas. Recuperado el 5 de junio de 2020 de: https:/ / naturvation.eu/nbs / barcelona/roof-top-garden-school-ferran-sunyer. 
Naturvation (2018a). Rehabilitación de un páramo industrial en un parque. Urban Nature Atlas. Recuperado el 2 de febrero de 2020 de: https:/ / naturvation.eu/nbs/marseille/rehabilitation-industrialwasteland-park.

Naturvation (2018b). Sistema de drenaje sostenible. Parque de Joan Reventós. Urban Nature Atlas. Recuperado el 5 de junio de 2020 de: https:/ / naturvation.eu/nbs/barcelona/sustainable-drainage-systems-suds-park-joan-reventos.

Naturvation (2018c). Proyecto de los 100000 árboles. Urban Nature Atlas. Recuperado el 5 de junio de 2020 de: https:/ / naturvation.eu/nbs/ porto/100000-trees-project.

Naturvation (2018d). Corredor verde urbano. Paseo de Sant Joan. Urban Nature Atlas. Recuperado el 5 de junio de 2020 de: https:/ / naturvation.eu/nbs/ barcelona/urban-green-corridor.

Naturvation (2018e). Residencia Nueva Naturaleza por el arquitecto Jean Nouvel. Urban Nature Atlas. Recuperado el 5 de junio de 2020 de: https: / / naturvation.eu/nbs/marseille/residence-new-naturejean-nouvel-architect.

Oltra, Christian y Marín, Renato (2013). Los retos en la adaptación al cambio climático en entornos urbanos. Papers: Revista de sociología, 98(2), 311-330. DOI: http:/ /dx.doi.org/10.5565/rev/papers/ v98n2.498.

Oppla (2018a). Barcelona: Soluciones basadas en la naturaleza (SbN) que mejoran la resiliencia al cambio climático. Oppla. Recuperado el 5 de junio de 2020 de: https://oppla.eu/casestudy/117.

Oppla (2018b). Lisboa: Soluciones basadas en la naturaleza $(S B N)$ que mejorar la resiliencia a través de la regeneración urbana. Desafios. Oppla. Recuperado el 5 de junio de 2020 de: https:/ / oppla.eu/lisbon-nature- based-solutions-nbs-enhancing-resilience-throughurban-regeneration.

Oppla (2020). Montpellier, Francia: Agroforestería: ¿agricultura del futuro? El caso de Montpellie. Oppla. Recuperado el 2 de febrero de 2021 de: https:/ / oppla. eu/casestudy/18469.

Sarabi, Shahryar Ershad, Han, Qi, Romme, A. Georges L., de Vries, Bauke y Wendling, Laura (2019). Key enablers of and barriers to the up take and implementation of nature-based solutions in urban settings: a review. Resources, 8(3), 121. DOI: https:// doi.org/10.3390/resources8030121.

Somarakis, Giorgios, Stagakis, Stavros y ChrysoulakisNektarios (Eds.) (2019). Think Nature Nature-Based Solutions Handbook. ThinkNature project funded by the EU Horizon 2020 research and innovation programme. Tesalónica: Foundation for Research and Technology - Hellas, FORTH. Recuperado el 30 de junio de 2020 de: http://oa.upm.es/35571/7/FEMP_Medidas_CCC_Planeamiento_urbano.pdf.

Verdaguer Viana-Cárdenas, Carlos, Fariña-Tojo, José, de Luxan-García de Diego, Margarita, Gómez-Muñoz, Gloria, Román López, Emilia, Velázquez-Valoria, Isabela y Sanz-Alduán, Alfonso (2015). Medidas para la mitigación y la adaptación al cambio climático en el planeamiento urbano. Guía metodológica. Madrid: Red Española de Ciudades por el Clima/ Oficina Española de Cambio Climático del

Ministerio de Agricultura, Alimentación y Medio Ambiente. Recuperado el 30 de junio de 2020 de: http://oa.upm.es/35571/7/FEMP_Medidas_CCC_ Planeamiento_urbano.pdf.

Yin, Robert K. (2009). How to do better case studies. En Bickman, Leonard y Rog, Debra J. (Eds.), The SAGE handbook of applied social research methods (2. ${ }^{\mathrm{a}}$ Edición) (pp. 254-282).Thousand Oaks: SAGE Pubblications.

Rey Mellado, Raquel, del Pozo Sánchez, Cristina y Franchini Alonso, María Teresa (2021). Soluciones basadas en la Naturaleza: estrategias urbanas para la adaptación al cambio climático. Hábitat y Sociedad, 14, 243-262.

<https://doi.org/10.12795/HabitatySociedad.2021.i14.13> 